\title{
Chronic Illness Diagnosis Helper: proposta de uma ferramenta para auxílio ao diagnóstico de doenças crônicas através da análise histórica de relatos sintomáticos
}

Chronic Illness Diagnosis Helper: proposal of a tool to aid the diagnosis of chronic diseases through the historical analysis of symptomatic reports

\section{Michael Lopes Bastos ${ }^{1}$ (D) https://orcid.org/0000-0001-6581-2067}

\author{
Anthony Lins ${ }^{2}$ (D) https://orcid.org/0000-0002-7153-841X \\ ${ }^{1}$ Escola Politécnica de Pernambuco, Universidade de Pernambuco, Recife, Brasil, \\ ${ }^{2}$ Departamento de Jogos Digitais, Universidade Católica de Pernambuco, Pernambuco, Brasil, \\ E-mail do autor principal: Michael Lopes Bastos mlb@poli.br
}

\section{Resumo}

Segundo dados da Organização Mundial da saúde (OMS), as doenças crônicas não transmissíveis (DCNT) são responsáveis por cerca de $71 \%$ dos óbitos em todo o mundo. Desse modo, ao longo dos anos algumas medidas vêm sendo tomadas para tentar reduzir esse índice. No que diz respeito ao uso de tecnologias nesse processo, existem algumas iniciativas no contexto do Aprendizado de Máquina (AM) que tentam encontrar formas que vão desde o auxílio ao diagnóstico até o suporte em determinados tipos de tratamentos. Visando isso, esse projeto tem como intuito apresentar uma ferramenta, baseada em um modelo de aprendizado de máquina, para auxiliar profissionais da saúde no diagnóstico das DCNT usando dados sintomáticos derivados da base "Chronic illness" da plataforma Kaggle. Como melhor resultado desse processo, foi escolhido um modelo de aprendizado baseado em técnicas de ensemble, onde a melhor precisão obtida chegou a $\approx 71,63 \%$ para um número de 20 patologias, sendo esse modelo usado como base para a aplicação Chronic Illness Diagnosis Helper (CIDH), desenvolvida para uma prova de conceito inicial.

Palavras-Chave: Doenças Crônicas; Inteligência Artificial; Auxílio ao diagnóstico;

\begin{abstract}
According to data from the World Health Organization (WHO), the noncommunicable chronic diseases (NCD) are responsible for around $71 \%$ of deaths in all world. Thus, over the years some methods have been taken to try to reduce this index. Concerning to use of Technologies in this process, there are some initiatives in the context of Machine Learning (ML) that trying to find ways from diagnosis aid to support in certain types of treatments. Thus, this project has a goal to show a tool based on a machine learning model to health professionals to diagnosis NCD using symptomatic data derivates from base "Chronic illness" from the Kaggle platform. As the best result from this process, was choose a learning model based an ensemble technics, when the best accurate arrived at $\approx 71,63 \%$ for some 20 pathologies, being this model used as bases to the application Chronic IIIness Diagnosis Helper (CIDH), developed with an initial Prove of Concept.
\end{abstract}

Key-words: Chronic diseases; Artificial Intelligence; Diagnostic aid. 


\section{INTRODUÇÃO}

As doenças crônicas ou doenças crônicas não transmissíveis (DCNT) segundo o estudo "Saving lives, spending less: a strategic response to NCDs" feito em 2018 pela Organização Mundial da Saúde (OMS), são responsáveis pela morte de cerca de 41 milhões de pessoas por ano, correspondendo a aproximadamente $71 \%$ dos óbitos da população mundial. Dentre todas essas ocorrências, 15 milhões são de pessoas com idade entre 30 e 69 anos e mais de $85 \%$ dos casos ocorrem em países de média e baixa renda mundial [1].

No Brasil, segundo o Ministério da Saúde, em 2015 essas doenças foram responsáveis pela morte de $51,6 \%$ da população que possuía de 30 a 69 anos de idade. De uma forma geral, as DCNT são chamadas de multifatoriais, ou seja, são acarretadas por uma série de fatores, sejam eles sociais ou individuais. A grande maioria dessas doenças possuem quatro fatores de risco em comum, são eles: o tabagismo, atividade física insuficiente, alimentação não saudável e o uso nocivo do álcool [2].

No que diz respeito ao uso de tecnologias nesse processo, existem algumas iniciativas no contexto do Aprendizado de Máquina (AM) que tentam encontrar formas que vão desde o auxílio ao diagnóstico até o suporte em determinados tipos de tratamentos [3]. Entretanto, a grande maioria dos estudos são voltados a doenças crônicas específicas, como doenças cardiovasculares [4], doenças arteriais coronárias [5] e doenças renais crônicas [6], por exemplo. Todos esses projetos aplicam técnicas de Inteligência Artificial (IA) como método base para chegar as soluções pretendidas.

Contudo, ainda existem muitas lacunas que podem ser preenchidas no que diz respeito ao uso das técnicas de IA no contexto das DCNT. O tratamento e diagnóstico médico ainda enfrentam uma série de dificuldades em relação a análise do grande volume de dados que existem atualmente. Os prejuízos e malefícios que as doenças crônicas trazem à saúde das pessoas é algo preocupante e aumenta continuamente [3].

Dessa forma, esse projeto tem como objetivo desenvolver uma ferramenta, baseada em um modelo de aprendizado de máquina, para auxiliar profissionais da saúde no diagnóstico das DCNT, tendo como base relatos sintomáticos de pacientes.

\section{MÉTOdOS}

Para o desenvolvimento do projeto, a base escolhida foi a "Chronic illness: symptoms, treatments and triggers" disponível no site Kaggle, uma comunidade online de cientistas de dados gerenciada pela Google LLC. Esse conjunto de dados é alimentado por informações cadastradas de pacientes com diferentes tipos de doenças crônicas em diferentes países do mundo. A coleta é feita a partir da plataforma Flaredown, um sistema voltado para obtenção de informações de sintomas, alimentação, clima, medicamentos e tratamentos de pessoas com esses tipos de patologias.

O processo metodológico desse trabalho é baseado em conjunto de três módulos, cada um possuindo uma série de passos sequenciais e iterativos, fazendo uso das técnicas e métodos necessários para chegar até a versão final da ferramenta proposta. A divisão dos módulos é feita de acordo com a natureza do processo, onde a primeira etapa é denominada Manipulação de dados, por possuir as atividades principais relacionadas a manipulação das informações da base de dados escolhida. O segundo módulo é descrito como Definição da ferramenta, pois trata de todo o processo de concepção e ideação para o desenvolvimento da prova de conceito (do inglês Proof of Concept - PoC) sugerida para o trabalho. A última etapa do processo é intitulada Desenvolvimento, pois lida com a parte da implementação dos conceitos definidos nas etapas anteriores. A Figura 1 demonstra um fluxograma geral desses módulos e etapas do processo.

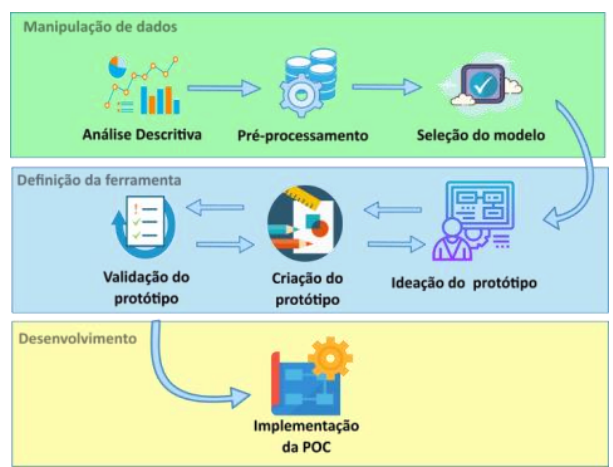

Figura 1: Processo metodológico para concepção final da ferramenta. Fonte: Próprio autor.

O módulo de Manipulação de dados é iniciado com a realização da Análise descritiva das informações da base de dados. Essa etapa é 
realizada para entender melhor como os dados estão dispostos e quais as abordagens devem ser levadas em consideração na etapa seguinte, o préprocessamento das informações. Esse ponto da fase de manipulação é um dos mais delicados e demorados do módulo. Nele, é feita uma reestruturação de várias colunas da base, remoção de valores inválidos e, seleção dos campos considerados mais importantes para o processo de classificação.

Visando a construção de um bom classificador, duas atividades complementares são desenvolvidas. Primeiro são implementados modelos de aprendizado de máquina convencionais, com o KNN e MLP. Depois é realizado um Ensemble com um conjunto heterogêneo de classificadores (etapa de seleção de modelos). Ao fim, os resultados são comparados e o melhor modelo é definido.

Após a seleção do modelo, é iniciada a etapa de Definição da ferramenta. Nesse ponto é realizada a ideação, criação e validação do protótipo do sistema.

Tendo a base da aplicação criada e validade, é dado início ao processo de implementação, que é a união do modelo de aprendizado de máquina criado com a codificação do protótipo concebido no módulo de Definição da ferramenta.

\section{RESULTADOS}

Para o desenvolvimento desta seção, serão abordados os resultados obtidos em cada uma das etapas do processo metodológico. Da subseção 3.1 até a 3.3 serão abordados os aspectos referentes a etapa de manipulação de dados. A subseção 3.4 relata as informações referentes ao processo de definição da ferramenta. Por fim, a subseção 3.5 explica a etapa de criação da prova de conceito final

\subsection{Análise Descritiva}

Para melhor entender as informações contidas da base "Chronic illness", foi necessário fazer uma exploração descritiva das informações existentes. Inicialmente, a base continha um conjunto de 9 colunas e 3.487 .633 registros armazenados. $O$ Quadro 1 representa a descrição de cada uma das colunas contidas na base.

Quadro 1: Descrição das características principais da base

\begin{tabular}{|l|l|}
\hline Coluna & Descrição \\
\hline user_id & $\begin{array}{l}\text { Se refere ao identificador único dos pacientes, } \\
\text { armazenado em um formato de string. }\end{array}$ \\
\hline age & Relata a idade de cada paciente. \\
\hline sex & Retrata um conjunto de quatro valores para \\
\hline
\end{tabular}

\begin{tabular}{|c|c|}
\hline & sexo: "female", "male", "other" e "doesnt_say". \\
\hline country & $\begin{array}{l}\text { Descreve a sigla do país ao qual o paciente está } \\
\text { imputando a informação. Ex.: 'BR' }\end{array}$ \\
\hline checkin_date & $\begin{array}{l}\text { Registra a data no formato AAAA-MM-DD em } \\
\text { que o paciente registrou a informação. }\end{array}$ \\
\hline trackable_type & $\begin{array}{l}\text { Se refere a } 7 \text { tipos diferentes de informações } \\
\text { inseridas pelo usuário, são elas: Symptom } \\
\text { (1.571.134 amostras), Weather (627.480 } \\
\text { amostras), Condition (505.461 amostras), } \\
\text { Treatment ( } 383.201 \text { amostras), Tag (207.902 } \\
\text { amostras), Food ( } 192360 \text { amostras) e HBI (95 } \\
\text { amostras). O tipo Condition se refere a } \\
\text { patologia já diagnosticada do paciente, } \\
\text { podendo existir mais de uma para o mesmo } \\
\text { paciente. O tipo Tag relaciona fatores } \\
\text { cotidianos que acontecem com os pacientes, se } \\
\text { ele bebeu ou dormiu mal na noite anterior, por } \\
\text { exemplo. Já o tipo HBI se refere ao Harvey } \\
\text { Bradshaw Index, uma medida padronizada para } \\
\text { avalizar a gravidade da doença de Crohn, } \\
\text { especialmente. }\end{array}$ \\
\hline trackable_ & $\begin{array}{l}\text { Essa coluna descreve os tipos de cada } \\
\text { trackable_type, ou seja, o nome de cada } \\
\text { Condition, Treatment, Tag e de todos os outros } \\
\text { elementos do tackable_type. }\end{array}$ \\
\hline trackable_id & $\begin{array}{l}\text { Demonstra um valor inteiro relativo ao } \\
\text { identificador único para cada "trackable_name" }\end{array}$ \\
\hline trackable_value & 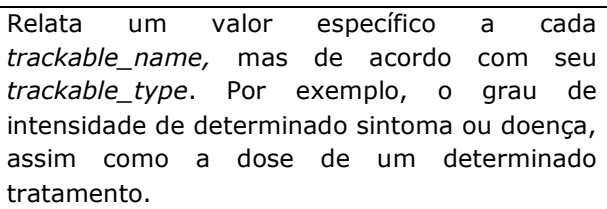 \\
\hline
\end{tabular}

Fonte: Próprio autor

Tendo em vista essas descrições, nota-se que várias informações importantes para futuras análises estão subdivididas em três principais colunas, a trackable_type, trackable_name e trackable_value, onde dividem informações a respeito de 7 categorias distintas, podendo cada uma ter uma coluna específica, se dividindo apenas entre seus nomes e valores. Visto essa estrutura inicial, foi necessário realizar a reestruturação da base através da criação de novas colunas e agrupamento de usuários, descritos na subseção a seguir.

\subsection{Pré-processamento}

Tendo em vista a má distribuição das informações observadas na análise descritiva, se fez necessário modificar a estrutura original da base. No caso em questão, o conteúdo da coluna trackable_type foi transformado em novas dimensões, logo, foram criadas as colunas Condition, Symptom, Treatment, Weather, Tag, Food e HBI. Seguindo a mesma lógica, também foram criadas colunas referentes aos valores mapeados na coluna trackable_value, sendo elas: Condition_value, Symptom_value, 


\section{Chronic Illness Diagnosis Helper: proposta de uma ferramenta para auxílio ao diagnóstico de}

doenças crônicas através da análise histórica de relatos sintomáticos

Treatment_value e Weather_value. As colunas Tag, Food e HBI não possuem um valor específico associado aos seus registros.

Para essa nova estrutura, além da criação das novas dimensões, foram feitas remoções de registros com valores NaN, com idades superiores a 117 anos e menores que 0 . Também foram realizados agrupamentos utilizando as colunas user_id e checkin_date, para pegar os registros de cada paciente a cada dia. Isso assegura, por exemplo, que determinado sintoma foi registrado no mesmo dia de determinada doença, ou que o mesmo sintoma esteja relacionado a algum fator cotidiano. A Figura 2 ilustra a transição da base inicial para sua primeira modificação.

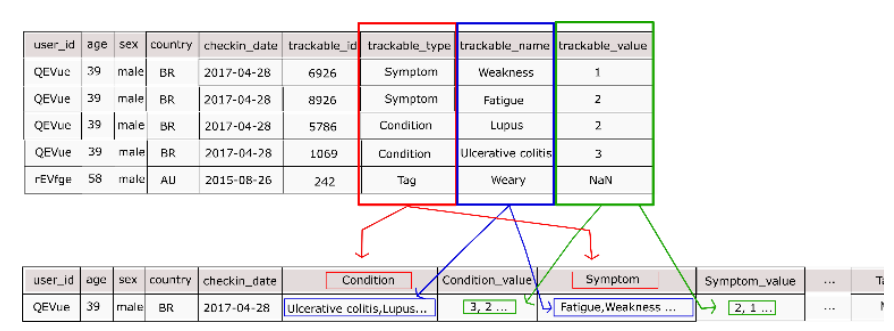

Figura 2: Reestruturação inicial da base Chronic illness. Fonte: Próprio autor.

Após esse procedimento, a base foi separada em 6 dataframes distintos, cada uma possuindo o valor referente a um dos trackable_type da base anterior, agrupando as informações do paciente (id, idade, sexo, data de inserção e país) ao trackable_type com o valor "Condition", que agora será utilizado como coluna de target para futuro uso em classificações supervisionadas.

Por conseguinte, para a construção do modelo, se decidiu utilizar os dados referentes aos sintomas dos pacientes, por estarem diretamente relacionados as principais características descritivas das patologias relatadas.

Com o dataframe apenas com as informações dos sintomas agrupadas, surgiu a necessidade de separar as informações dos sintomas de cada paciente, pois agora existia uma lista de sintomas em uma mesma coluna, podendo trazer complicações no momento da inserção das informações em um futuro processo de classificação.

Por esse motivo, foi criado uma nova coluna com o nome dos primeiros 250 sintomas mais relatados entre os pacientes. Algumas variações dessa quantidade de colunas foram realizadas, mas por limitações de hardware, 250 colunas foi o maior valor suportada para o novo formato do dataframe.

Com as novas colunas já criadas, foi adicionado o valor 1 quando o sintoma estava relacionado ao paciente e o valor 0 quando esse valor não estava entre sua lista de sintomas e, depois do agrupamento já realizado, foram eliminadas as colunas user_id, checkin_date e country, consideradas desnecessárias para o processo de predição. O resultado final da base de sintomas é representado de forma resumida na Figura 3.

\begin{tabular}{|lr|l|c|c|c|c|c|}
\hline age & sex & Condition & Condition_value & Fatigue & Headache & Nausea & Joint pain \\
\hline 39 & male & Lupus & 4 & 1 & 0 & 0 & 0 \\
\hline 39 & male & Lupus & 4 & 1 & 0 & 0 & 1 \\
\hline 39 & male & Lupus & 4 & 1 & 0 & 0 & 1 \\
\hline 39 & male & Migraine & 0 & 0 & 0 & 0 & 1 \\
58 & male & Diabetes & 1 & 0 & 0 & 0 & 1 \\
\hline
\end{tabular}

Figura 3: Resultado do pré-processamento da base de dados, utilizando apenas os valores dos sintomas. Fonte: Próprio autor.

Além do exposto, houve também um processo de normalização e balanceamento das informações através do recurso Categorical da biblioteca pandas (utilizada para transformar o campo sexo em valores numéricos), factorize (utilizado para fazer uma rotulação e categorização dos targets), StandardScaler e o Normalizer do sklearn.preprocessing, para escalonar e normalizar o restante dos dados [7]. Para balancear os dados foi utilizado um processo denominado de Oversampling, deixando as amostras com quantidades equivalentes, evitando uma futura classificação tendenciosa para as classes com maior representatividade [8].

Por fim, a base resultado de todo esse processo ficou com 254 colunas e um conjunto de 300.884 instâncias relativas ao cruzamento dos sintomas com cada patologia. Dessa forma, as informações se encontram melhor estruturadas para o uso em futuras tentativas de classificação ou agrupamento de novas informações

\subsection{Seleção do modelo}

Para o processo de seleção do modelo foram testados dois tipos diferentes de abordagens. A primeira utilizando modelos de aprendizado de máquina [9] com o KNN e MLP e, a segunda utilizando várias técnicas de ensemble a partir de um conjunto heterogêneo de classificadores [10]. O melhor resultado é selecionado e utilizado como 
modelo final. Nos tópicos seguintes, os modelos testados são detalhados.

\subsubsection{Multilayer Perceptron (MLP)}

A MLP [11] é um tipo de modelo que tem sido bastante utilizado em problemas de auxílio ao diagnóstico de doenças, como relata Setsirichok et al. [12] e possui um bom comportamento em problemas de múltiplas classes, segundo o mesmo autor.

Para o problema em questão, a rede criada possui 4 vezes a dimensão de colunas da base - 1 ((input_dim*4) -1) e 4 camadas escondidas com 128 neurônios cada uma, utilizando a relu como função de ativação. Esses parâmetros foram definidos através de um conjunto inicial de testes. Com uma estrutura de camadas definida, foram testadas 4 combinações de épocas e batch size, cada uma executada por 10 vezes (Tabela 1 ).

Tabela 1: Acurácia e taxa de perda de variadas configurações de épocas e batch size para MLP com 10 classes

\begin{tabular}{c|c|c|c}
\hline $\begin{array}{c}\text { Número de } \\
\text { Épocas }\end{array}$ & Batch Size & Taxa de perda & Acurácia \\
\hline 20.000 & 1.000 .000 & {$[0,09714625]$} & {$[0,71127867]$} \\
\hline 6.000 & 20.000 & {$[\mathbf{0 , 0 7 8 4 0 3 0 6}]$} & {$[0,72679924]$} \\
\hline 1.000 & 10.000 & {$[0,10821427]$} & {$[0,69504005]$} \\
\hline 500 & 5.000 & {$[0,11439618]$} & {$[\mathbf{0 , 7 3 2 7 4 2 3 5}]$}
\end{tabular}

Fonte: Próprio autor

Analisando os dois melhores resultados, através da visualização dos gráficos da Figura 4 percebe-se que o modelo do gráfico ' $A$ ' acaba entrando em overfitting $^{1}$ e se ajusta muito rápido ao processo de aprendizado, sendo uma abordagem a ser descartada para a seleção do modelo final. Por conseguinte, de todas as alternativas testadas a com 500 épocas e batch size de 5.000 , obteve a melhor média da acurácia, com aproximadamente 73,27\%.
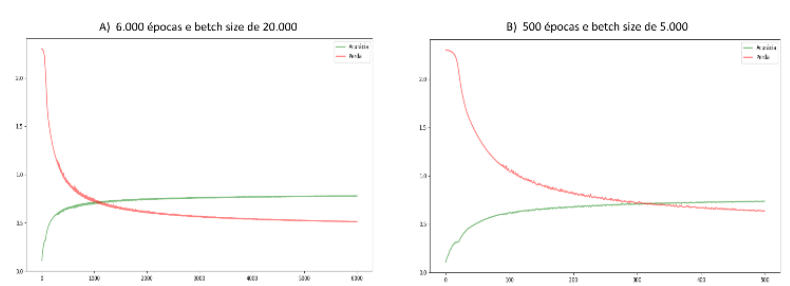

Figura 4: Gráfico de convergência dos dois melhores resultados da MLP. Fonte: Próprio autor.

\subsubsection{K-nearest neighbors (KNN) classifier}

O KNN realiza seu processo de identificação de uma classe desconhecida a partir do voto da maioria dos vizinhos mais próximos [13].

Nesse projeto, todos os parâmetros desse modelo foram definidos por meio da execução de um algoritmo genético ( $A G$ ), onde o mesmo buscou encontrar a melhor combinação dos parâmetros por meio de um processo baseado na escolha da melhor solução [14] utilizando a acurácia do modelo como função objetivo e variando os parâmetros relativos ao número de vizinhos (n_neighbors ou K) e o tipo de algoritmo utilizado (algorithm). Como resultado da seleção dos parâmetros, a melhor solução definiu o n_neighbors $=2$ (escolhendo de 1 a 10) e o algorithm = 'kd_tree' (escolhendo entre 'ball_tree', 'kd_tree', 'brute' e 'auto').

Para a evolução do modelo e divisão do conjunto de dados em treinamento e teste foram utilizadas técnicas bastante comuns em aprendizado de máquina, como o TrainTestSplit e a validação por meio de $K$-folds cross validation [15]. Seguindo o padrão de execução da MLP (3.3.1), foram executadas 10 repetições com TrainTestSplit e 10 utilizando cross validation, variando o $\mathrm{K}$-fold em 4,7 e 10, como é detalhado na Tabela 2.

Tabela 2: Resultados da média de 10 iterações do TrainTestSplit e Cross Validation com variação de K-Fold de 4, 7 e 10 para o KNN

\begin{tabular}{|c|c|c|}
\hline Divisão de base & Desvio Padrão & Acurácia \\
\hline TrainTestSplit & {$[0,00310232]$} & {$[0,66935870]$} \\
\hline $\begin{array}{c}\text { Cross Validation: } \\
\text { K-Fold }=4\end{array}$ & {$[0,00133886]$} & {$[0,67268614]$} \\
\hline $\begin{array}{c}\text { Cross Validation: } \\
\text { K-Fold }=7\end{array}$ & {$[0,00104294]$} & {$[0,67855559]$} \\
\hline $\begin{array}{c}\text { Cross Validation: } \\
\text { K-Fold }=10\end{array}$ & {$[0,00096968]$} & {$[\mathbf{0 , 6 8 0 6 6 2 9 0 ]}$} \\
\hline
\end{tabular}

Fonte: Próprio autor.
1 Quando o modelo usa abordagens mais complicadas que o necessário [22]. 


\section{Chronic Illness Diagnosis Helper: proposta de uma ferramenta para auxílio ao diagnóstico de}

doenças crônicas através da análise histórica de relatos sintomáticos

\subsubsection{Métodos Ensemble}

Segundo Chaudhary et al. [16] ensembles (veja mais sobre ensembles em [10]) vêm sendo bastante recomendados para problemas de classificação de várias classes. Tendo em vista isso, foram feitos vários testes utilizando diferentes tipos de ensembles e diferentes quantidades de classes para o problema em questão.

Inicialmente, um conjunto de 10 classificadores foram executados separadamente na base de dados utilizando seus parâmetros padrão. Dentre esses, os 4 que obtiveram a melhor acurácia foram selecionados (RandomForestClassifier, KNeighborsClassifier, ExtraTreesClassifier e DecisionTreeClassifier) para formar a base dos classificadores do ensemble. O primeiro método executado foi o Max Voting, que realiza uma votação entre as saídas de cada classificador utilizado, tendo como saída a classe "mais votada" entre os classificadores base [17].
Em uma segunda abordagem de ensemble, foi utilizando o método Staking. Ele utiliza o resultado da base de modelos do ensemble (camada 1) como entrada para um modelo final (camada 2) [18]. Nesse tocante, surge as outras duas técnicas de ensemble utilizadas, o Bagging e o Boosting (para ler mais sobre, veja [18]).

Com base nessas informações, nesse projeto tanto o Boosting como o Bagging foram utilizados como modelos finais (camada 2) do Staking, com os mesmos classificadores base do Max Voting (camada 1). Desse modo, dois foram os classificadores utilizados para os testes com Boosting, o XGBoostClassifier e o CatBoostClassifier [19]. Para os testes com Bagging foi utilizado apenas 0 RandomForestClassifier. A

Tabela 3 demonstra a média e desvio padrão da acurácia para 10 iterações utilizando $20,10,5,3$ e 2 classes para cada abordagem escolhida.

Tabela 3: Resultados de 10 iterações para os ensembles dos 4 melhores classificadores utilizando diferentes números de classes

\begin{tabular}{|c|c|c|c|c|c|c|}
\hline Método & Classificadores & $\begin{array}{c}\text { Acurácia para } 20 \\
\text { classes } \\
\text { Média } \\
\text { (Desvio Padrão) }\end{array}$ & $\begin{array}{l}\text { Acurácia para } 10 \\
\text { classes } \\
\text { Média } \\
\text { (Desvio Padrão) }\end{array}$ & $\begin{array}{c}\text { Acurácia para } 5 \\
\text { classes } \\
\text { Média } \\
\text { (Desvio Padrão) }\end{array}$ & $\begin{array}{l}\text { Acurácia para } 3 \\
\text { classes } \\
\text { Média } \\
\text { (Desvio Padrão) }\end{array}$ & $\begin{array}{c}\text { Acurácia para } 2 \\
\text { classes } \\
\text { Média } \\
\text { (Desvio Padrão) }\end{array}$ \\
\hline Max Voting & $\begin{array}{c}\text { Random Forest } \\
\text { KNeighborsClassifier } \\
\text { ExtraTreesClassifier } \\
\text { DecisionTreeClassifier }\end{array}$ & $\begin{array}{c}0,71455093 \\
(0,00015715)\end{array}$ & $\begin{array}{c}0,73728597 \\
(0,00037731)\end{array}$ & $\begin{array}{r}0,70891455 \\
(0,00062014)\end{array}$ & $\begin{array}{c}0,67871588 \\
(0,00115697)\end{array}$ & $\begin{array}{c}0,85081484 \\
(0,00114085)\end{array}$ \\
\hline Staking & ExtraTreesClassifier & $\begin{array}{c}0,71540214 \\
(0,00000000) \\
\end{array}$ & $\begin{array}{c}0,73717798 \\
(0,00000000) \\
\end{array}$ & $\begin{array}{c}0,71037543 \\
(0,00000000) \\
\end{array}$ & $\begin{array}{c}0,68302899 \\
(0,00000000)\end{array}$ & $\begin{array}{r}\mathbf{0 , 8 5 1 5 6 0 9 7} \\
(0,00000000) \\
\end{array}$ \\
\hline $\begin{array}{c}\text { Staking } \\
\text { /Bagging } \\
\end{array}$ & Radom Forest & $\begin{array}{c}0,71514687 \\
(0,00000000) \\
\end{array}$ & $\begin{array}{c}0,73778668 \\
(0,00000000) \\
\end{array}$ & $\begin{array}{c}0,71127867 \\
(0,00000000) \\
\end{array}$ & $\begin{array}{c}0,68237450 \\
(0,00000000) \\
\end{array}$ & $\begin{array}{l}\mathbf{0 , 8 5 1 5 6 0 9 7} \\
(0,00000000) \\
\end{array}$ \\
\hline \multirow{2}{*}{$\begin{array}{l}\text { Staking } \\
\text { /Boosting }\end{array}$} & XGBoostCla & $\begin{array}{r}\mathbf{0 , 7 1 6 3 2 5 0 1} \\
(0,00000000)\end{array}$ & $\begin{array}{c}0,73870955 \\
(0,00000000)\end{array}$ & $\begin{array}{c}0,71159284 \\
(0,00000000)\end{array}$ & $\begin{array}{r}\mathbf{0 , 6 8 3 0 9 4 4 4} \\
(0,00000000)\end{array}$ & $\begin{array}{r}\mathbf{0 , 8 5 1 5 6 0 9 7} \\
(0,00000000)\end{array}$ \\
\hline & CatBoostClassifier & $\begin{array}{c}0,71160265 \\
(0,00000000)\end{array}$ & $\begin{array}{r}\mathbf{0 , 7 3 8 8 6 6 6 4} \\
(0,00000000)\end{array}$ & $\begin{array}{r}\mathbf{0 , 7 1 3 0 0 6 6 0} \\
(0,00000000)\end{array}$ & $\begin{array}{c}0,68289810 \\
(0,00000000)\end{array}$ & $\begin{array}{r}\mathbf{0 , 8 5 1 5 6 0 9 7} \\
(0,00000000)\end{array}$ \\
\hline
\end{tabular}

Fonte: Próprio autor.

Dado a variação dos valores médios das acurácias para o conjunto de testes com mais de 3 classes, notou-se que os classificadores poderiam estar se confundindo muito em relação a algumas classes em específico, visto que ele sobe de $\approx 68 \%$ nos testes com 3 classes para $\approx 85 \%$ nos testes com 2 classes, variando bastantes nos testes anteriores. Desse modo, foi gerado uma matriz de confusão (Figura 5) dos resultados obtidos com os esperados (targets) para melhor entender o comportamento dos classificadores. 


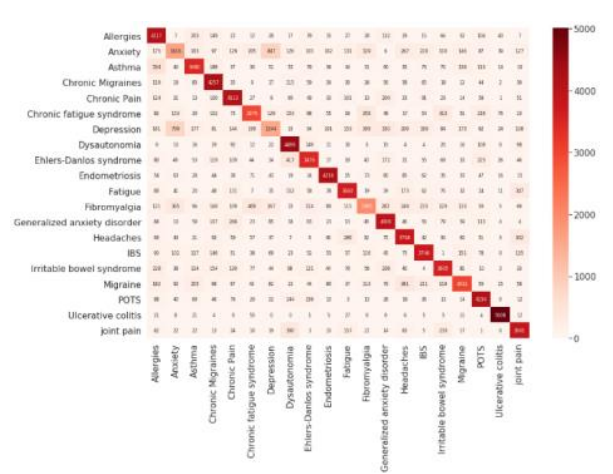

Figura 5: Matriz de confusão para o ensemble com 20 classes utilizando o XGBoostClassifier como classificador final do Staking. Fonte: Próprio autor.

Analisando a matriz de confusão, nota-se frequência mais alta de erros do classificador ao tentar realizar a predição das classes "Depressão" e "Ansiedade", podendo esse ser o motivo da volatilidade dos resultados para as diferentes quantidades de classes, visto que a escolha das classes é feita por sua maior representatividade na base, onde "Depressão" e "Ansiedade" são a segunda e terceira com maior número de amostras.
A fim de evitar esse problema, foi criado uma nova base, agora com a união dos dados referentes a ansiedade e depressão, transformando-os em uma única classe. $O$ resultado dessa nova abordagem é demonstrado na Tabela 4.

De acordo com os novos resultados, é perceptível uma normalidade maior em relação à média da acurácia para seu conjunto de classes, tendo em vista que, quanto maior o número de classes mais difícil será o processo de predição para o classificador. Essa nova abordagem também melhorou bastante a média geral da acurácia para cada conjunto de testes, crescendo em mais de $10 \%$ em alguns casos.

\subsection{Chronic Illness Diagnosis Helper (CIDH): definição da ferramenta}

A construção da prova de conceito foi inspirada em alguns dos aspectos do Design Thinking, desenvolvido por Tim Brown [20]. O método proposto por Brown

Tabela 4: Resultados de 10 iterações unindo as classes depressão e ansiedade para os ensembles dos 4 melhores classificadores utilizando diferentes números de classes.

\begin{tabular}{|c|c|c|c|c|c|c|}
\hline Método & Classificadores & $\begin{array}{c}\text { Acurácia para } 20 \\
\text { classes } \\
\text { Média } \\
\text { (Desvio Padrão) }\end{array}$ & $\begin{array}{c}\text { Acurácia para } 10 \\
\text { classes } \\
\text { Média } \\
\text { (Desvio Padrão) }\end{array}$ & $\begin{array}{l}\text { Acurácia para } 5 \\
\text { classes } \\
\text { Média } \\
\text { (Desvio Padrão) }\end{array}$ & $\begin{array}{l}\text { Acurácia para } 3 \\
\text { classes } \\
\text { Média } \\
\text { (Desvio Padrão) }\end{array}$ & $\begin{array}{c}\text { Acurácia para } 2 \\
\text { classes } \\
\text { Média } \\
\text { (Desvio Padrão) }\end{array}$ \\
\hline Max Voting & $\begin{array}{c}\text { Random Forest } \\
\text { KNeighborsClassifier } \\
\text { ExtraTreesClassifier } \\
\text { DecisionTreeClassifier }\end{array}$ & $\begin{array}{c}0,76304991 \\
(0,00009863)\end{array}$ & $\begin{array}{c}0,80166618 \\
(0,00018383)\end{array}$ & $\begin{array}{c}0,83909919 \\
(0,00060233)\end{array}$ & $\begin{array}{c}0,83039136 \\
(0,00048337)\end{array}$ & $\begin{array}{c}0,86534925 \\
(0,00063078)\end{array}$ \\
\hline Staking & ExtraTreesClassifier & $\begin{array}{c}0,76339764 \\
(0,00000000)\end{array}$ & $\begin{array}{c}0,80298158 \\
(0,00000000)\end{array}$ & $\begin{array}{c}0,84112833 \\
(0,00000000)\end{array}$ & $\begin{array}{c}0,83290029 \\
(0,00000000)\end{array}$ & $\begin{array}{l}\mathbf{0 , 8 6 6 8 7 7 7 8} \\
(0,00000000)\end{array}$ \\
\hline $\begin{array}{l}\text { Staking } \\
\text { /Bagging }\end{array}$ & Radom Forest & $\begin{array}{c}0,76321495 \\
(0,00000000)\end{array}$ & $\begin{array}{c}0,80292069 \\
(0,00000000)\end{array}$ & $\begin{array}{c}0,84117704 \\
(0,00000000)\end{array}$ & $\begin{array}{l}\mathbf{0 , 8 3 3 1 0 3 2 8} \\
(0,00000000)\end{array}$ & $\begin{array}{c}\mathbf{0 , 8 6 6 8 7 7 7 8} \\
(0,00000000)\end{array}$ \\
\hline \multirow{2}{*}{$\begin{array}{l}\text { Staking } \\
\text { /Boosting }\end{array}$} & XGBoostClassifier & $\begin{array}{c}0,76379348 \\
(0,00000000) \\
\end{array}$ & $\begin{array}{c}0,80310338 \\
(0,00000000)\end{array}$ & $\begin{array}{c}0,84151807 \\
(0,00000000) \\
\end{array}$ & $\begin{array}{c}0,83298149 \\
(0,00000000) \\
\end{array}$ & $\begin{array}{l}\mathbf{0 , 8 6 6 8 7 7 7 8} \\
(0,00000000)\end{array}$ \\
\hline & CatBoostClassifier & $\begin{array}{l}\mathbf{0 , 7 6 5 7 9 0 9 5} \\
(0,00000000)\end{array}$ & $\begin{array}{l}\mathbf{0 , 8 0 3 6 2 7 1 1} \\
(0,00000000)\end{array}$ & $\begin{array}{c}\mathbf{0 , 8 4 1 9 0 7 8 2} \\
(0,00000000)\end{array}$ & $\begin{array}{c}0,83294089 \\
(0,00000000)\end{array}$ & $\begin{array}{c}0,86626880 \\
(0,00000000)\end{array}$ \\
\hline
\end{tabular}

Fonte: Próprio autor.

aborda o design centrado no ser humano, de tal forma que sua preocupação inicial deve ser explorar as necessidades do usuário e, apenas depois criar o design do produto. Esse processo segue um conjunto de três passos principais, a "inspiração" a "ideação" e por fim, a "implementação" da ferramenta [21].

Para esse projeto, a fase de "inspiração" é explorada na introdução desse documento, através das justificativas para atacar o problema da dificuldade do diagnóstico das DCNT e, a consequente necessidade da criação de uma ferramenta que pudesse auxiliar na redução do tempo levado nesse processo. A etapa de ideação foi realizada por meio da criação de um Mockup, validado por um conjunto de profissionais de computação. A fase de "implementação" será abordada no tópico a seguir.

\subsection{Implementação da ferramenta}

O desenvolvimento da PoC final foi iniciado tendo como foco plataformas web, implementada com base no framework Django e utilizando Bootstrap, DOI: 10.25286/repa.v5i2.1354 


\section{Chronic Illness Diagnosis Helper: proposta de uma ferramenta para auxílio ao diagnóstico de}

doenças crônicas através da análise histórica de relatos sintomáticos

JavaScript, HTML e CSS como ferramentas para manipulação de suas propriedades gráficas. Todo layout foi criado com a preocupação de manter um nível adequado de responsividade sobre as telas.

Em relação a base de dados, a aplicação contém as entidades usuarios, sintomas, tem_paciente, tem_sintomas e pacientes. Dentre todas as entidades, as que de fato estão sendo utilizadas no momento são sintomas e usuarios, armazenando os 250 sintomas utilizados como colunas da base tratada e, os dados dos médicos que utilizaram a aplicação, respectivamente.

Para as telas da aplicação, a implementação foi feita com base no Mockup e nas sugestões dos profissionais que participaram do processo de validação. A Figura 6 (A) demonstra como ficou a tela inicial. Na Figura 6 (B) é demonstrada a tela de registro de informações sobre o paciente.

Sugerido no processo de validação, a tabela de fatores cotidianos foi substituída, dando espaço a um campo interativo de pesquisa e uma listagem livre dos sintomas selecionados. Ao fim da página existe também um botão para dar início ao processo de predição do classificador. A tela de loading utilizada durante a execução desse processo Figura 6(C) contém um gif com os ícones utilizados na logo da ferramenta.

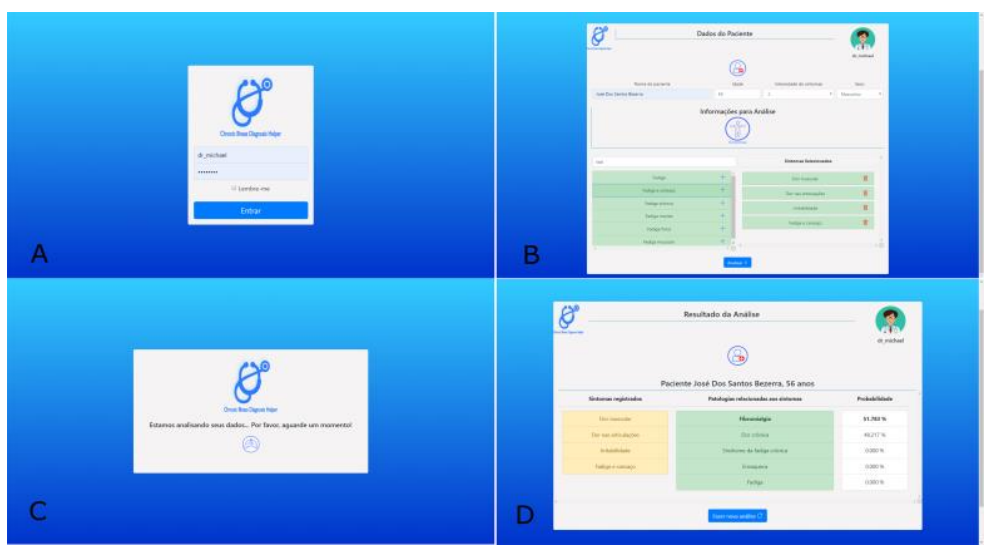

Figura 6: Conjunto de telas da aplicação. Fonte: Próprio autor.

Por fim, na Figura 6 (D) está a tela com os resultados da análise feita pelo modelo, relatando além das doenças e da probabilidade de cada uma, os sintomas utilizados como base para a predição. Ao fim da página existe a opção de solicitar uma nova análise e, no canto superior esquerdo das telas de resultado e registro de pacientes, há um ícone de um médico, indicando a possibilidade de sair do sistema.

\section{DISCUSSÕES}

Tendo em vista as três principais fases do projeto, algumas considerações podem ser feitas a respeito dos resultados obtidos por cada uma. $\mathrm{Na}$ etapa de manipulação dos dados houve um grande esforço para que os dados pudessem ser organizados de modo a proporcionar um conjunto de dados viável para uma futura classificação. O fato das principais informações relativas as patologias dos pacientes estarem todas em uma só coluna, dificultou bastante o processo de cruzamento das informações.
Nesse sentido, o ideal seria que cada amostra contivesse todas as informações relativas a coluna trackable_type da tabela, ou seja, de uma única vez o paciente inserisse informações sobre o tipo de sua doença, quais sintomas estariam relacionados àquela doença, quais fatores (tags) interferiram na intensidade dos sintomas, alimentação e clima, por exemplo, todos em uma única amostra.

No caso de tentar realizar essa união para a base em questão, haveria uma grande perda de informações. Grande parte das amostras seriam eliminadas, podendo trazer prejuízos para o processo de classificação, tendo em vista também o crescimento das dimensões do problema.

$\mathrm{Na}$ seleção dos modelos, cada abordagem foi testada com 10 iterações, salvando ao fim a média e desvio padrão da acurácia de cada uma. A Figura 7 demostra os resultados referentes a cada modelo, todos relativos a base com 10 classes 


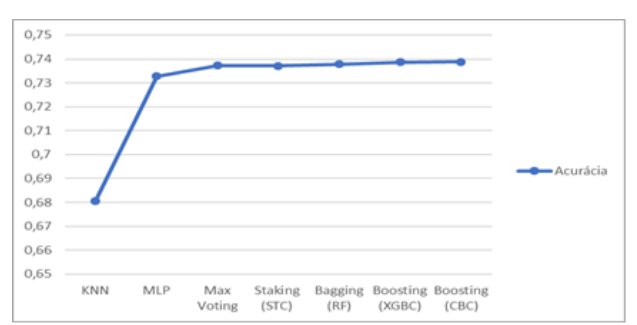

Figura 7: Média da acurácia de todos os modelos testados para 10 classes. Fonte: Próprio autor.

Fica perceptível uma certa similaridade entre as médias finais de cada modelo, ficando apenas o KNN um pouco abaixo dos demais. Visto que os melhores resultados foram os ensembles, mais especificamente 0 Boosting utilizando CatBoostClassifier com $\approx 78,88 \%$, uma nova abordagem foi testada para identificar possíveis melhorias para o processo de classificação.

$\mathrm{Na}$ primeira abordagem, foi identificado através da análise da matriz de confusão, uma tendência no processo de classificação. Haviam muitos erros dos modelos ao tentar diferenciar as classes "Depressão" e "Ansiedade" e, por esse motivo, elas foram unidas e os modelos foram novamente treinados com essa nova configuração. De acordo com as duas abordagens, com e sem a união das classes, percebe-se um crescimento de mais de $10 \%$ em alguns casos, sendo o melhor resultado para duas classes, obtidos pelo ExtraTreesClassifier, RandomForest e XGBoostClassifier com $\approx 86,68 \%$ de precisão.

Todavia, devido ao ensemble utilizar vários classificadores para obter sua predição, o tamanho do modelo final fica muito grande, acima de $2 \mathrm{~Gb}$ (no caso de 10 classes) e acima de 4Gb (no caso de 20 classes), aumentando significativamente o tamanho do projeto. Em contrapartida, o MLP com maior acurácia $(\approx 73,27 \%)$ não chega a $5 \mathrm{Mb}$, podendo ser uma melhor alternativa dependendo da infraestrutura existente para implantação da ferramenta. O uso ou não dos modelos com as classes unidas traria maior precisão para as predições, entretanto, esse tipo de informação deve ser analisado por um especialista de saúde.

Por fim, a PoC foi desenvolvida, sendo ajustada conforme algumas sugestões dos avaliadores e com a viabilidade de implementação de algumas funcionalidades.

\section{CONCLUSÕES}

Nesse projeto foi proposto uma nova abordagem, com base na criação de uma ferramenta, para dar suporte a profissionais de saúde no auxílio ao diagnóstico de doenças crônicas por meio de um modelo de aprendizado de máquina aplicado a dados sintomáticos de pacientes.

Durante todo o processo de desenvolvimento foram encontradas algumas dificuldades, em relação a tempo, hardware e a desestruturação da base para com os objetivos principais do trabalho. Entende-se que, com a estrutura necessária e com tempo abio, técnicas como grid search ou algoritmos genéticos poderiam ser utilizados para encontrar uma melhor combinação de parâmetros para os modelos desenvolvidos.

Outra possibilidade para a base seria o uso de algoritmos não supervisionados, na tentativa de encontrar os melhores agrupamentos para as patologias. Os mesmos também poderiam ser utilizados para separar essas patologias em subgrupos (doenças crônicas cardíacas, reumatológicas, etc.) e, a partir deles, uma classificação mais específica poderia ser realizada, tentando predizer um grupo menor de doenças em áreas mais especializadas.

Para trabalhos futuros, pretende-se desenvolver um novo módulo para a aplicação, que terá como objetivo fazer o registro de todas as informações relatadas pela base utilizada, porém, de maneira mais estruturada e separando as patologias por área de especialização, já pensando na construção de um novo modelo de classificação que possa ser ainda mais eficiente que o desenvolvido neste projeto, tudo isso sendo adaptado também para dispositivos móveis.

Visto o apresentado, fica evidente que um vasto esforço foi realizado para a obtenção de um conjunto positivo de resultados, tanto no desenvolvimento do modelo de classificação quanto na implementação da aplicação final, atingindo os objetivos iniciais do projeto. Todavia, entende-se que ainda existe um grande leque de oportunidades em aberto, possibilitando novas pesquisas e o desenvolvimento de soluções cada vez mais eficientes.

\section{AGRADECIMENTOS}

Queria agradecer a minha família, amigos e minha namorada Andressa, por me darem os 
motivos que preciso para alcançar todos os objetivos da minha vida. Aos meus colegas de residência que deram imensas contribuições para que esse trabalho pudesse ser desenvolvido. Ao meu orientador Anthony Lins e ao professor Carmelo Bastos pelas grandes colaborações e dicas e, as instituições FITec/SECTI/CMA-Parqtel/UPE/FACEPE por incentivar o desenvolvimento profissional e tecnológico no estado por meio de projetos como esse.

\section{Referências}

[1] World Health Organization., Saving lives, spending less A strategic response to noncommunicable diseases, p. 20, 2018.

[2] Ministério da Saúde, Vigilância de Doenças Crônicas Não Transmissíveis (DCNT), $2018 . \quad$ [Online]. Available: http://www.saude.gov.br/vigilancia-emsaude/vigilancia-de-doencas-cronicas-naotransmissiveis-dcnt. [Accessed: 22-Aug2019].

[3] HUANG, M. J. ;CHEN, M. Y.; LEE, S. C. Integrating data mining with case-based reasoning for chronic diseases prognosis and diagnosis, Expert Syst. Appl., vol. 32, no. 3, pp. 856-867, 2007.

[4] MEZZATESTA, $S$. et al. A machine learningbased approach for predicting the outbreak of cardiovascular diseases in patients on dialysis, Comput. Methods Programs Biomed., vol. 177, pp. 9-15, 2019.

[5] ABDAR, M. et al. A new machine learning technique for an accurate diagnosis of coronary artery disease, Comput. Methods Programs Biomed., vol. 179, p. 104992, 2019.

[6] ALMANSOUR N. A. et al., Neural network and support vector machine for the prediction of chronic kidney disease: A comparative study, Comput. Biol. Med., vol. 109, no. April, pp. 101-111, 2019.

[7] Singh, D.; Singh, B. Investigating the impact of data normalization on classification performance, Appl. Soft Comput. J., no. XXXX, p. 105524, 2019.

[8] KIM, Y. G; KWON, Y.; PAIK, M. C. Valid oversampling schemes to handle imbalance, Pattern Recognit. Lett., vol. 125, pp. 661-667, 2019.

[9] BATES, A.; SALDIAS, B. A comparison of machine learning and logistic regression in modelling the association of body condition score and submission rate,
Prev. Vet. Med., vol. 171, no. November 2018, p. 104765, 2019.

[10] HERBRICH, R.; GRAEPEL, T. Ensemble Methods, Foundations and Algorithms. Taylor \& Francis Group, LLC, Cambridge, UK, p. 232, 2012.

[11] GARDNER, M. W.; DORLING, S. R. Artificial neural networks (the multilayer perceptron) - a review of applications in the atmospheric sciences, Atmos. Environ., vol. 32, no. 14-15, pp. 2627-2636, 1998.

[12] SETSIRICHOK, D. et al., Classification of complete blood count and haemoglobin typing data by a C4.5 decision tree, a naïve Bayes classifier and a multilayer perceptron for thalassaemia screening, Biomed. Signal Process. Control, vol. 7, no. 2, pp. 202-212, 2012.

[13] PODDAR, M. G. et al. Automated Classification of Hypertension and Coronary Artery Disease Patients by PNN, KNN, and SVM Classifiers Using HRV Analysis. Elsevier Inc., 2019.

[14] PARINAM, S. et al. An improved optical parameter optimisation approach using Taguchi and genetic algorithm for high transmission optical filter design, Optik (Stuttg)., vol. 182, pp. 382-392, 2019.

[15] KAJÓ, M.; NOVÁCZKI, S. A Genetic Feature Selection Algorithm for Anomaly Classification in Mobile Networks, Proc. 19th Int. ICIN Conf.-Innov. Clouds, Int. Netw., pp. 204-211, 2016.

[16] CHAUDHARY, A.; KOLHE, S.; KAMAL, R. A hybrid ensemble for classification in multiclass datasets: An application to oilseed disease dataset, Comput. Electron. Agric., vol. 124, pp. 65-72, 2016.

[17] HOSNI, $M$. Reviewing ensemble classification methods in breast cancer, Comput. Methods Programs Biomed., vol. 177, pp. 89-112, 2019.

[18] RIBEIRO, M. H. D. M.; COELHO, L. S. Ensemble approach based on bagging, boosting and stacking for short-term prediction in agribusiness time series, Appl. Soft Comput. J., p. 105837, 2019.

[19] HUANG G. et al., Evaluation of CatBoost method for prediction of reference evapotranspiration in humid regions, $J$. Hydrol., vol. 574, no. December 2018, pp. 1029-1041, 2019.

[20] BROWN, T. Change by design:How Design Thinking Transforms Organizations and Inspires Innovation. HarperBusiness, 2009.

[21] Bastos, M. L. CatalogToMakers: uma plataforma de catalogação colaborativa 
de componentes eletrônicos e projetos de computação física, Universidade Federal de Pernambuco, 2019.

[22] HAWKINS, D. M. The Problem of Overfitting, J. Chem. Inf. Comput. Sci., vol. 44, no. 1, pp. 1-12, 2004. 